The International Journal of Multimedia \& Its Applications (IJMA) Vol.4, No.3, June 2012

\title{
MEDiCAL IMAgE SEgMENTATION BY MARKER- CONTROLLED WATERSHED AND MATHEMATICAL MORPHOLOGY
}

\author{
Ahmad EL ALLAOUI ${ }^{1}$ and M'barek NASRI $^{1}$ \\ ${ }^{1}$ LABO MATSI, ESTO, B.P 473, University Mohammed I OUJDA, MOROCCO. \\ ahmadallaoui@yahoo.fr ; nasridest.ump.ma
}

\begin{abstract}
Segmentation by watershed transform is a fast, robust and widely used in image processing and analysis, but it suffers from over-segmentation. We present in this paper some improvements to this algorithm based on the mathematical morphology in order to get over this difficulty. The performance of this method is validated on medical images. The results obtained show the good performance of this approach.
\end{abstract}

\section{KEYWORDS}

Image processing, medical image segmentation, watershed, Marker controlled watershed, reconstruction, dilatation, Mathematical morphology.

\section{INTRODUCTION}

The segmentation is a very important stage in images and interpretation processing. There are two main approaches to segmentation: the frontier approach and the region approach [13, 14]. The segmentation by watershed combine the two approaches $[1,2]$.This is a powerful technique for rapid detection of both edges and regions.

The major problem of the watershed transform is over-segmentation. Indeed, this algorithm is sensitive to any local minimum in the image, and tends to define the lines of the watershed transform where each local minimum gives rise to a region. To avoid this problem, powerful tools adapted to different problems have been proposed in the literature $[3,7]$ :

- Either reduce the number of minima and avoid calculation of too many regions.

- Proceed by either filtering techniques by merging the regions according to similarity criteria after spectral and spatial application of the watershed.

We have used markers to reduce the number of regional minima.

In Section 2 image segmentation with watershed algorithm is presented. The proposed method is presented in section 3. In section 4, a validation of our approach is given; experimental results are obtained by medical images. Finally we give a conclusion. 


\section{WATERSHED ALGORITHM}

The watershed transform is a tool morphological based for image segmentation. It is proposed by Digabel and Lantuejoul $[1,2,4,12]$, which consider a grey level image as a topographic relief. If one combines the grey level of each point at an altitude. It is then possible to define the watershed transform as the ridge forming the boundary between two watersheds. This is to compute the watershed of the said relief. Watersheds thus obtained correspond to regions of the image.

Watershed represents the boundaries between adjacent catchments. The minimum can be interpreted as markers of watershed regions and the watershed can be interpreted as contours, figure 1.

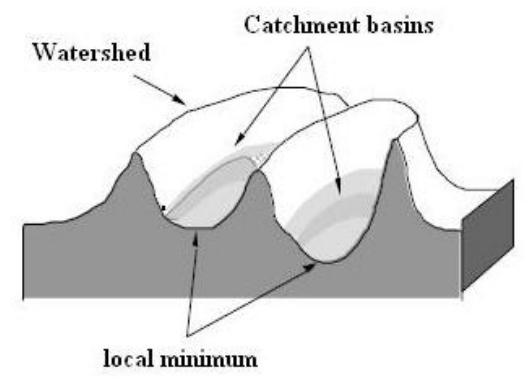

Figure 1: Modelling of contours by watershed transform

Many pre-processing techniques are proposed by the different researchers (filters, morphological operators) were then designed to reduce the number of regions not significant. Nevertheless, the work of Beucher [1,2] demonstrated the limitations of these methods namely the oversegmentation.

\subsection{The over-segmentation}

The use of the single watershed algorithm does not really allow good segmentation because far too many regions are detected, figure 2 . There are two main methods to limit this oversegmentation:

- hierarchical watershed segmentation $[1,2,9]$.

- and watershed by markers $[3,10,11]$.
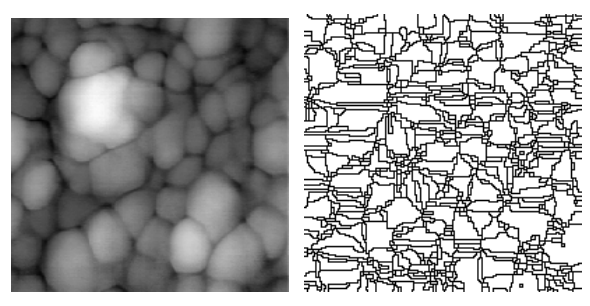

Original image Watershed 715 regions

Figure 2: Over-segmentation.

The hierarchical segmentation: The hierarchical approach is to generate a tree of regions from the result of the watershed. Regions and watershed are first indexed, and then the process of 
The International Journal of Multimedia \& Its Applications (IJMA) Vol.4, No.3, June 2012

hierarchical segmentation process merges the regions whose borders are the lowest. The result is a tree in which it is possible to explore the different levels of fusion regions $[1,2,6,8,9]$.

Several studies have been made to get over the problem of over-segmentation by markers $[1,3,5$, $7,10,11]$.

\subsection{Mathematical morphology}

Mathematical morphology is a nonlinear branch of the signal processing field and concerns the application of set theory concepts to image analysis. Morphology refers to the study of shapes and structures from a general scientific perspective. Morphological filters or operators are nonlinear transformations, which modify geometric features of images.

These operators transform the original image into another image through the iteration with other image of a certain shape and size which is known as structuring element [10].

The structuring element is a basic structure that will be used to analyze locally, by comparison, the shapes of interest.

The choice of shape and size of the structuring element is very important: it influences the result [10], it may take the form of a disk of radius $r$, a rectangle or other shape.

There are several tools morphological in image segmentation namely erosion, dilation, reconstruction etc. We are interested in this work in the morphological reconstruction based on dilatation.

\subsubsection{Morphological dilatation}

Let $X$ be a subset of $E$. If at any $x$ we associate a position $B(x)$ of the structuring element $B$, then the set $\mathrm{X}$ dilated by the structuring element $B$ is defined as following:

$$
\delta_{B}(X)=\{x \in E, B(x) \cap X \neq \phi\}
$$

The structuring element $B$, denoted by its center is moved to occupy successively all positions of the space $E$. For each position, we ask the question: does $B$ intersect at $X$ ? Positive responses are all dilated, figure 3.

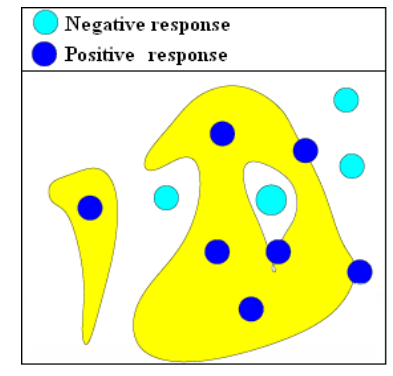

Figure 3: Morphological dilatation.

The dilatation is characterized by the following properties:

- Fills holes smaller than B.

- Fills narrow channels. 
- Welds both forms close.

- The size of objects increases.

- Small details disappear.

In what follows, we propose a watershed by markers based on morphological reconstruction.

\subsection{Morphological reconstruction}

The morphological reconstruction is well known in the case of binary images, where it simply extracts the connected components of an image, which are "marked" with another image. The extended grey scale reconstruction, it can perform multiple tasks such as image filtering, domes, and extraction of the basin. In this paper, we used the image reconstruction algorithm based on morphological dilation.

Morphological reconstruction is based on morphological dilation [6], but notes the following unique properties:

- Processing is based on two images, a marker and a mask, rather than one image and a structuring element.

- Processing is based on the concept of connectivity, rather than a structuring element.

- Processing repeats until stability; i.e., the image no longer changes.

Morphological reconstruction can be thought of conceptually as repeated dilations of an image, called the marker image, until the contour of the marker image fits under a second image, called the mask image. In morphological reconstruction, the peaks in the marker image "spread out," or dilate. Figure 4 illustrates this processing in 1-D.

The marker and the mask can be two intensity images or binary having the same size. The resulting image is an intensity image or binary $[4,6]$.

The marker must have the same size as the mask, and its elements must be less than or equal to the elements of the mask. Connectivity used is 8 -connected. [4, 6]

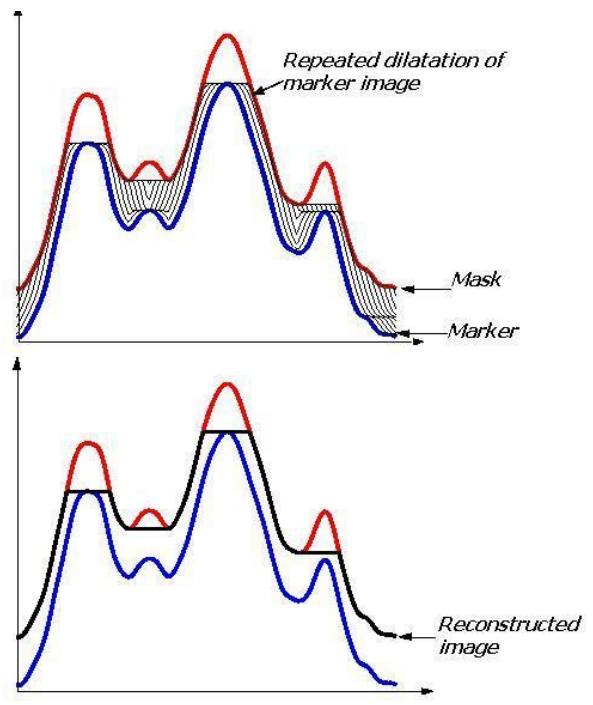

Figure 4: Repeated dilations of marker image, constrained by mask.[6] 
The International Journal of Multimedia \& Its Applications (IJMA) Vol.4, No.3, June 2012

To illustrate morphological reconstruction, consider this simple image $I$. It contains two primary regions, the blocks of pixels containing the values 14 and 18 . The background is primarily all set to 10 , with some pixels set to 11 .

$I=\quad \begin{array}{lllllllll}10 & 10 & 10 & 10 & 10 & 10 & 10 & 10 & 10 \\ 10 & 13 & 13 & 13 & 10 & 11 & 10 & 11 & 10 \\ 10 & 13 & 13 & 13 & 10 & 10 & 10 & 10 & 11 \\ 10 & 13 & 13 & 13 & 10 & 11 & 10 & 11 & 10 \\ 10 & 10 & 10 & 10 & 10 & 10 & 10 & 10 & 10 \\ 10 & 10 & 10 & 10 & 10 & 17 & 17 & 17 & 10 \\ 10 & 11 & 10 & 10 & 10 & 17 & 17 & 17 & 10 \\ 10 & 10 & 11 & 10 & 10 & 17 & 17 & 17 & 10 \\ 10 & 11 & 10 & 11 & 10 & 10 & 10 & 10 & 10 \\ 10 & 10 & 10 & 10 & 10 & 10 & 11 & 10 & 10\end{array}$

One way to create a marker image $M r$ is to subtract a constant $h$ from the mask image $I$, but we must determine this constant.

$$
M r=I-h
$$

The choice of the constant $h$ is very important, it depends on the processed image, and we must choose the right value of this constant.

In this example we chose $h=2$ then $\quad M r=I-2$

$\begin{array}{cccccccccc}8 & 8 & 8 & 8 & 8 & 8 & 8 & 8 & 8 \\ 8 & 11 & 11 & 11 & 8 & 9 & 8 & 9 & 8 \\ 8 & 11 & 11 & 11 & 8 & 8 & 8 & 8 & 9 \\ 8 & 11 & 11 & 11 & 8 & 9 & 8 & 9 & 8 \\ M_{r}=\quad & 8 & 8 & 8 & 8 & 8 & 8 & 8 & 8 & 8 \\ 8 & 9 & 8 & 8 & 8 & 15 & 15 & 15 & 8 \\ 8 & 8 & 9 & 8 & 8 & 15 & 15 & 15 & 8 \\ 8 & 9 & 8 & 9 & 8 & 15 & 15 & 15 & 8 \\ 8 & 8 & 8 & 8 & 8 & 8 & 8 & 8 & 8 \\ 8 & 8 & 8 & 8 & 8 & 8 & 9 & 8 & 8\end{array}$

Call the morphological reconstruction to reconstruct the image. In the output image $I_{r e}$, note how all the intensity fluctuations except the intensity peak have been removed [6].

In the output image, all insignificant local maxima will be deleted.

$I_{r e}=\begin{array}{lllllllll}10 & 10 & 10 & 10 & 10 & 10 & 10 & 10 & 10 \\ 10 & 11 & 11 & 11 & 10 & 10 & 10 & 10 & 10 \\ 10 & 11 & 11 & 11 & 10 & 10 & 10 & 10 & 10 \\ 10 & 11 & 11 & 11 & 10 & 10 & 10 & 10 & 10 \\ 10 & 10 & 10 & 10 & 10 & 10 & 10 & 10 & 10 \\ 10 & 10 & 10 & 10 & 10 & 15 & 15 & 15 & 10 \\ 10 & 10 & 10 & 10 & 10 & 15 & 15 & 15 & 10 \\ 10 & 10 & 10 & 10 & 10 & 15 & 15 & 15 & 10 \\ 10 & 10 & 10 & 10 & 10 & 10 & 10 & 10 & 10 \\ 10 & 10 & 10 & 10 & 10 & 10 & 10 & 10 & 10\end{array}$

\section{PROPOSED SEgMENTATION}

We have developed a segmentation method based on morphological operation, and watershed applied to grey level images. The key points of this method are:

- Morphological reconstruction 
The International Journal of Multimedia \& Its Applications (IJMA) Vol.4, No.3, June 2012

- Extract the markers of regions

- Application of watershed transform

The watershed is the separation lines of greatest intensity in an image.

The markers will define the sources from which the algorithm of the watershed will simulate the rising waters.

Figure 5 show the steps of the proposed method.

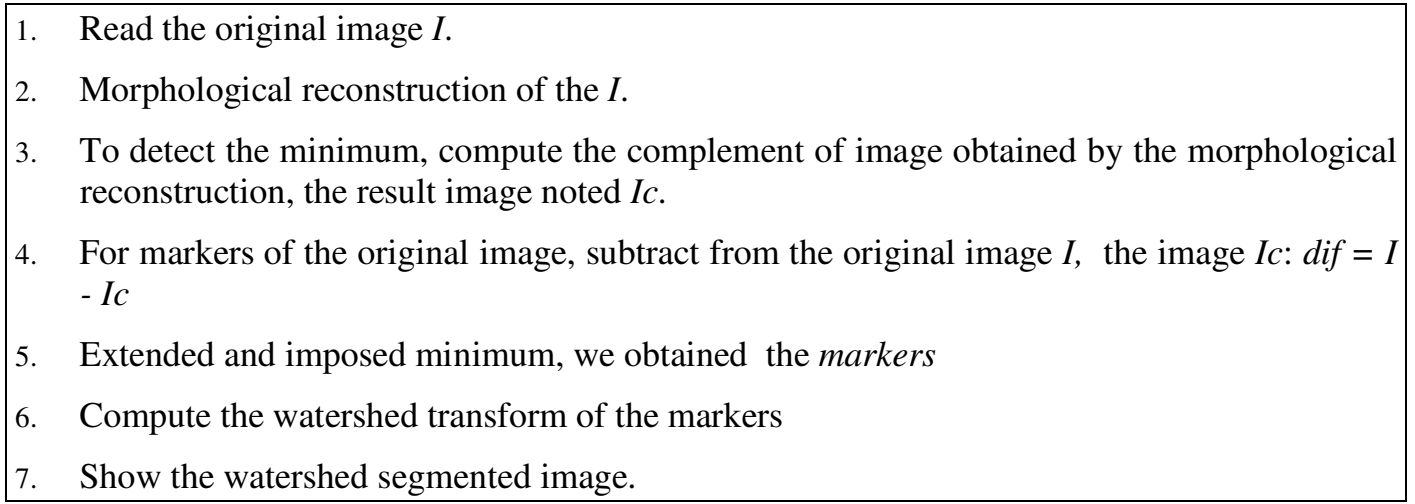

Figure 5: Proposed algorithm.

\section{EXPERIMENTALE RESULTS}

\subsection{Introduction}

In order to evaluate the performances of the proposed method we have considered 6 grey level medical images: II to I6. We have compared the results obtained by our method with those obtained by watershed without markers.

\subsection{Results of segmentation}

Figure 6 and table 1 show the results of segmentation.

a. initial image $a$,

b. watershed applied to the image $a$,

c. image markers

d. watershed applied to the image $c$

e. superposition of the initial image with watershed. 
The International Journal of Multimedia \& Its Applications (IJMA) Vol.4, No.3, June 2012

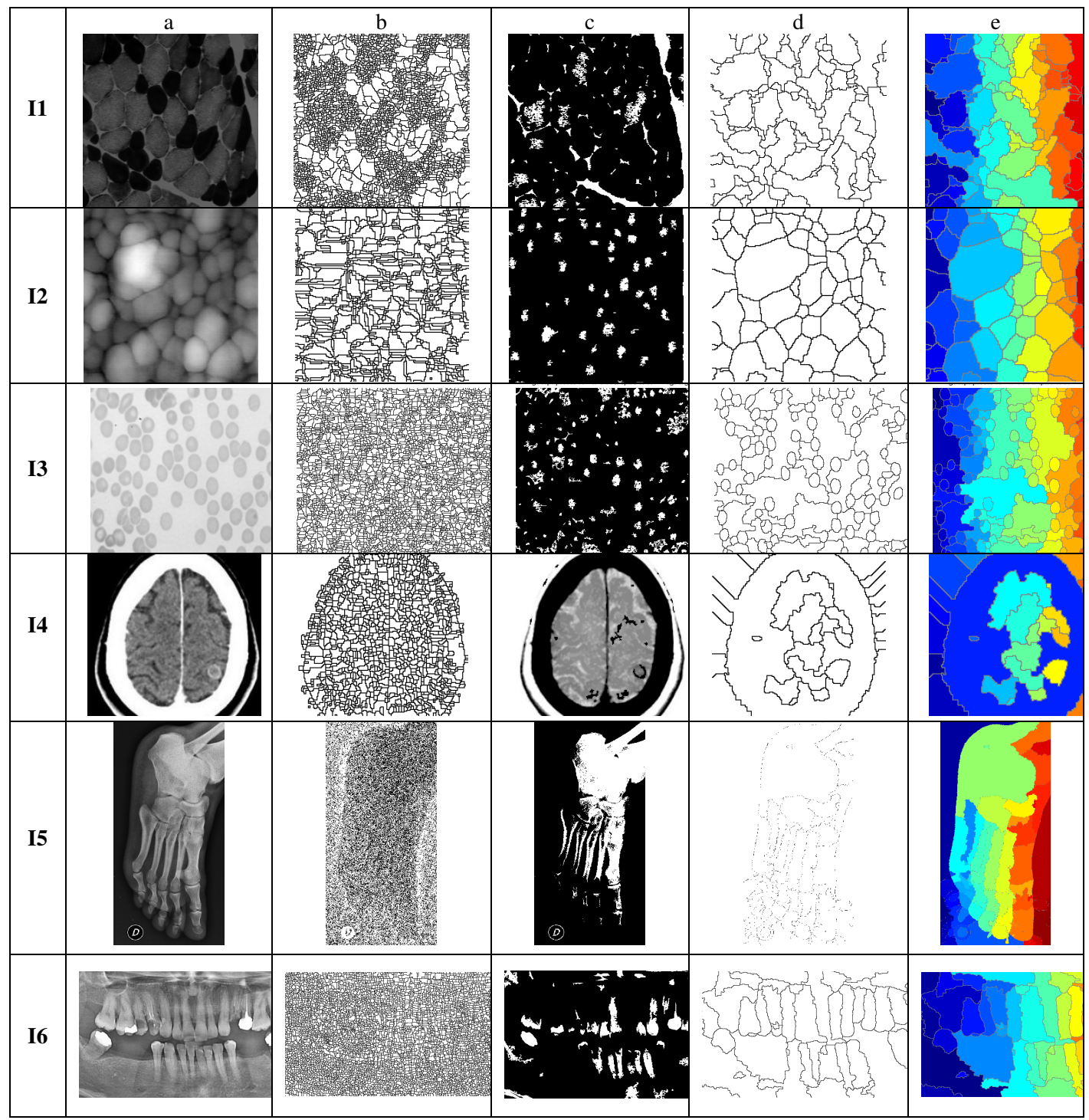

Figure 6: Results of segmentation.

The proposed method is applied to 6 medical images (X-ray, MRI) II to I6: cell, muscle, brain, foot and dental image. The results of segmentation by the proposed algorithm shows the clarity and detection of objects (region and contour) marked by the image markers $c$.

The result obtained by watershed without markers (Figure 6.b) gives no information on the regions of the original image. Against the result obtained by watershed with markers shows the speed of segmentation and no more than 2 seconds in all tests. Thus the proposed method detects all objects of the original image (Figure 6.d and 6.e).

In table 1, the number of regions obtained was decreased; the proposed value of the constant $h$ used in the reconstruction is well chosen and well the best. The problem of local minima is eliminated then the problem of over-segmentation is solved.

We proposed a simple algorithm, fast, easy to implement and get over the problem of oversegmentation. 
The International Journal of Multimedia \& Its Applications (IJMA) Vol.4, No.3, June 2012

The algorithm is useful to segment the objects that touch each other in an image.

Table1 : Information results

\begin{tabular}{|l|c|c|c|c|c|c|}
\hline Image & $I 1$ & $I 2$ & $I 3$ & $I 4$ & $I 5$ & $I 6$ \\
\hline Value of constant $\boldsymbol{h}$ & 14 & 5 & 3 & 30 & 47 & 45 \\
\hline $\begin{array}{l}\text { Number of regions without } \\
\text { markers }\end{array}$ & 2772 & 715 & 3253 & 1047 & 164503 & 8376 \\
\hline Number of regions with our method & 107 & 72 & 143 & 31 & 95 & 51 \\
\hline
\end{tabular}

\section{CONCLUSION}

In this paper, we have proposed a method of segmentation watershed by markers and morphological operation. It is able to segment real medicals images. We have calculated automatically region markers. The markers are used to control the watershed to obtain good results.

The markers are generated automatically positioned and thereby avoiding the problem of oversegmentation, and manual marking.

The experimental results obtained show the good performance of this approach. This approach may be used for problems of decision support in medical diagnosis.

\section{REFERENCES}

[1] S. Beucher, Unbiased Implementation of the Watershed Transformation based on Hierarchical Queues. CMM Internal note, Paris 206 School of Mines, 2004.

[2] Serge Beucher. Watershed, hierarchical segmentation and waterfall algorithm. Dans Mathematical morphology and its applications to image processing, pages 69-76. Kluwer Academic Publishers, 1994.

[3] M. Ali HAMDI. Modified Algorithm marker-controlled watershed transform for Image segmentation Based on Curvelet Threshold.Canadian Journal on Image Processing and Computer Vision. pp, 88-91. Vol. 2 No. 8, December 2011.

[4] J. Serra, Image Analysis and Mathematical Morphology. Academic Press, London 1982.

[5] R. C. Gonzalez and R. E. Woods, Digital Image Processing, Prentice-Hall, Upper Saddle River, NJ, USA, 2nd edition, 2002.

[6] Vincent, L., "Morphological Grayscale Reconstruction in Image Analysis: Applications and Efficient Algorithms," IEEE Transactions on Image Processing, Vol. 2, No. 2, pp. 176-201, April, 1993.

[7] Sarah Ghandour. Segmentation d'images couleurs par morphologie mathématique : application aux images microscopiques. Thèse doctorat, Université Paul Sabatier, Toulouse 12 Juillet 2010.

[8] P. Arbeláez and L. Cohen. Constrained image segmentation from hierarchical boundaries. CVPR, 2008.

[9] P. Arbeláez, M. Maire, C. Fowlkes and J. Malik.Contour Detection and Hierarchical Image Segmentation IEEE TPAMI, Vol. 33, No. 5, pp. 898-916, May 2011.

[10] K. Parvati, et al, Image Segmentation Using Gray-Scale Morphology and Marker-Controlled watershed Transformation. Discrete Dynamics in Nature and Society, vol. 2008, Article ID 384346, 8 pages, 2008.

[11] S. Lefèvres. Segmentation par Ligne de Partage des Eaux avec Marqueurs Spatiaux et Spectraux. XXIIe colloque GRETSI (traitement du signal et des images), Dijon (FRA), 8-11 septembre 2009. 
The International Journal of Multimedia \& Its Applications (IJMA) Vol.4, No.3, June 2012

[12] H. Digabel et C. Lantuejoul. Iterative algorithms. Dans 2nd European Symp. Quantitative Analysis of Microstructures in Material Science, pp. 85-99, Biology and Medicine. Caen, France, 1978.

[13] A. EL Allaoui, M. Merzougui, M. Nasri, M. EL Hitmy and H. Ouariachi. Evolutionary Image Segmentation By Pixel Classification Application To Medical Images. IJCIIS International Journal of Computational Intelligence and Information Security, ISSN: 1837-7823, Vol. 2, No. 3 pp. 12-24. March 2011.

[14] A. EL Allaoui, M. Nasri, M. Merzougui , M. EL Hitmy et B. Bouali. Medical Image Segmentation By Region Evolutionary Approach. The 3rd International Conference on Multimedia Computing and Systems ICMCS'12 Tangier, CDROM, 10-12 May 2012.

\section{Authors}

Ahmad EL ALLAOUI works on image processing and pattern recognition in MATSI laboratory of the school of Technology, University of Oujda, Morocco. He has his master in the field of computer sciences at University the Med Benabdellah at Fez. His main field of research interest is segmentation of medical image based on new methods (genetic and evolutionary approaches, neural networks, fuzzy logic).

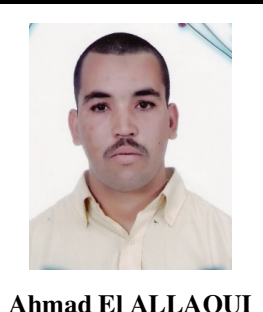

M'barek Nasri is a senior lecturer at the School of technology of Oujda University since 1996. He obtained his Ph.D degree in 2004 in the field of data Classification from the University of Oujda, department of physics. He obtained the habilitation degree in 2006 from the same university. His field of research interest is in image processing and its application to the quality control by artificial vision and medical imaging. MATSI laboratory of the school of Technology, University of Oujda, Morocco

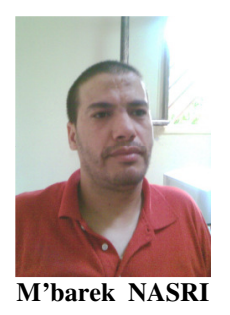

